\title{
Beschreibung der landschaftsökologischen Raumgliederung Europas - Teil 2
}

\author{
Inga Weustermann · Gunther Schmidt · Winfried Schröder
}

Erhalten: 7. Februar 2008/Akzeptiert: 11. September 2008/Online veröffentlicht: 29. Oktober 2008

(C) Springer-Verlag 2008

Zusammenfassung Hintergrund und Ziel Die Raumgliederung für Europa (ELCE) mit 40 Klassen wurde in Teil 1 dieser Beitragsserie (Hornsmann et al. 2008) mit dem Klassifikationsverfahren Classification and Regression Trees (CART) berechnet. Sie soll als Instrument zur räumlichen Optimierung europäischer Umweltmessnetze dienen. In diesem zweiten Teil der Serie werden die Datengrundlagen und die Raumklassen der ELCE näher betrachtet. Zudem wird die Einhaltung des statistischen Qualitätskriteriums Trennschärfe untersucht.

Material und Methoden Der ELCE liegen Karten zur potenziell natürlichen Vegetation (PNV), zur Bodenart, zur Höhe und zum Klima zugrunde. Neben der Beschreibung der Eingangskarten wird die Aufbereitung der Daten für die Berechnungen mit CART und das Verfahren selbst erläutert. Die ELCE-Raumklassen können hinsichtlich der zugrunde liegenden ökologischen Daten qualitativ und quantitativ beschrieben werden: Mit Hilfe von Kreuztabellen wurden die nominalen Daten ausgewertet. Für die metrischen Merkmale wurden deskriptiv statistische Kennwerte bestimmt und mit Histogrammen, Boxplots, Jahresgangkurven zum Klima oder

Herausgeber: Gunther Schmidt · Winfried Schröder

Vorwort (Schmidt und Schröder 2008)

Teil 1: Berechnung einer landschaftsökologischen Raumgliederung Europas (Hornsmann et al. 2008)

Teil 2: Beschreibung der landschaftsökologischen Raumgliederung Europas (Weustermann et al.)

Teil 3: Vergleich der landschaftsökologischen Raumgliederung Europas mit bestehenden Raumgliederungen

Teil 4: Repräsentanzanalyse für ökologische Langzeitmessprogramme in Europa

I. Weustermann $(\square) \cdot$ G. Schmidt $\cdot$ W. Schröder

Lehrstuhl für Landschaftsökologie, Hochschule Vechta, PF 1553,

49364 Vechta, Deutschland

E-Mail: iweustermann@iuw.uni-vechta.de mit Klimadiagrammen veranschaulicht. Über die Einhaltung des statistischen Qualitätskriteriums Trennschärfe für die metrischen Merkmale (Klimaelemente, Höhe) der Raumklassen der ELCE mit 40 Klassen gab der U-Test Aufschluss.

Ergebnisse Drei der flächengrößten 40 Raumklassen der ELCE werden in einem tabellarischen Überblick hinsichtlich ihrer räumlichen Lage und häufigsten PNV-Kartierungseinheiten gekennzeichnet. Die ökologischen Eigenschaften dieser Klassen werden ausführlich im Text beschrieben. Der U-Test ergab, dass in $97 \%$ der Fälle für die Höhen- und monatlichen Klimadaten je zweier Raumklassen der 40er Gliederung sehr signifikante Unterschiede ihrer Verteilungen vorlagen.

Diskussion Die drei Raumklassen der ELCE zeigen deutlich unterschiedliche landschaftsökologische Ausprägungen. Die Ergebnisse des U-Tests bestätigen diese Beobachtung: Nur in drei Prozent aller Fälle liegen keine signifikanten Unterschiede vor. Dies ist darauf zurückzuführen, dass der U-Test besonders auf Unterschiede bei den Medianen von Verteilungen reagiert. In den Fällen ohne signifikantes Ergebnis glichen sich die Medianwerte der Höhen- oder monatlichen Klimawerte zwischen den einzelnen Raumklassen. In diesen Fällen sind aber die Kennwerte der Verteilungen anderer Eingangsgrößen signifikant unterschiedlich.

Schlussfolgerungen Die Wahl der Eingangskarten, die Vorgehensweise bei der Vorbereitung der Daten und das Verfahren zur Klassifikation der Landschaftsausschnitte mit Hilfe von CART haben sich bewährt. Die 40 ökologischen Raumklassen der ELCE erfüllen das statistische Qualitätskriterium der Trennschärfe. Diese ökologische Raumgliederung Europas ist für die Repräsentanzanalyse europaweiter Umweltmonitoringmessnetze und als räumliche Bezugsgrundlage in einem WebGIS aufgrund der transparenten Methodik und der mit dessen Hilfe differenziert statistisch beschreibbaren Raumklassen gut geeignet. 
Empfehlungen Die ELCE sollte für die Analyse und Bewertung derjenigen Umweltmessnetze genutzt werden, deren Daten für die Beschreibung und Bewertung des Umweltzustands in Europa zusammengeführt und ausgewertet werden können. In diesem Zusammenhang ist eine Einbindung der Raumgliederung sowie bestimmter Messnetze samt ihrer Metadaten in ein WebGIS von Vorteil, da hierdurch ein schneller Zugriff auf die Daten und eine integrierende Auswertung mit Daten anderer Messnetze und weiteren flächenhaften Informationen zum Umweltzustand oder zur Landnutzung ermöglicht werden.

Ausblick In Teil 3 dieser Serie wird ein Vergleich zu alternativen Landschaftsgliederungen Europas auf verschiedenen Maßstabsstufen durchgeführt. Ein vierter Beitrag soll Repräsentanzanalysen für einige europäische Umweltmessnetze präsentieren.

Schlüsselwörter Classification and Regression Trees $($ CART) · Europäische Raumgliederung · Klassifizierung · Ökologische Raumklassen · Potenziell natürliche Vegetation (PNV) · Repräsentanz · Trennschärfe · Umweltmonitoring

\section{Description of the ecological land classification of Europe}

\begin{abstract}
Background, aim, and scope As described in article 1 of this series (Hornsmann et al. 2008), the ecological land classification of Europe (ELCE) encompassing 40 classes was calculated by using Classification and Regression Trees (CART). Amongst other applications, ELCE could be used as a basis for the spatial optimisation of European environment monitoring networks. This second article of the series deals with the data used for the calculation of ELCE. In addition, the derived ecological classes were described according to their environmental properties. Finally, the compliance with the quality criteria on "discrimination" was checked.

Materials and methods ELCE was calculated by using maps on the potential natural vegetation (PNV), soil texture, elevation and climatic elements. Besides the description of these base maps, the pre-processing of these data as well as the CART algorithm are explained. All the ecological classes of ELCE can be described quantitatively and qualitatively regarding their ecological characteristics. By means of cross tabulation the categorical characteristics for each class of ELCE were determined. For the metric characteristics the medians for each class and for Europe were computed. Histograms, box plots and curves on the annual course of climate or climate charts were used to illustrate these findings. The method to assess the adherence of the quality criteria for the metrical variables of the ELCE40 version was the U-test.
\end{abstract}

Results In a table the geographic location and the most frequent PNV mapping units covered by three exemplary ecoregions of the 40 classes of ELCE are compiled. The ecological characteristics of these classes are described in the text. Concerning the U-test, in $97 \%$ of the pair-wise comparisons between two classes there are significant distinctions between the distributions of the altitude and monthly climate data of each two classes of the regionalisation in 40 classes.

Discussion Three of the largest ecoregions clearly show different ecological characteristics. The results of the U-test confirm this even if, however, 3 percent of the comparisons did not reveal significant differences. This is due to the fact that the U-test is mainly sensible to differences in the median of the distributions. In the cases without a significant result the medians of the metrical characteristics of two classes are similar to each other, whereas other statistical values of distribution differ significantly.

Conclusions The chosen input data, their preparation and the calculation of the ELCE proved their worth. The quality criterion on discrimination is met by the ELCE with forty classes. The ELCE is suitable for the test of representativity of European networks and as a spatial framework of a WebGIS on environmental monitoring in Europe because of its transparent derivation and its statistically and ecologically well-discriminated ecoregions.

Recommendations The ecological land classification should be used for the assessment of monitoring networks from which the data are compiled for evaluations of the environmental condition in Europe. In this context, the implementation of the regionalisation and the measurement networks in a WebGIS could be useful because it allows fast data access and an integrative analysis of related environmental measurements as well as other surface maps on environmental and landuse properties.

Perspectives In part 3 of this series, the ELCE will be compared with existing ecoregionalisations of Europe on different spatial scales. The fourth article will focus on the investigation of the representativity of some environmental European monitoring networks.

Keywords Classification and regression trees (CART) Discrimination · Ecological classes · Ecoregionalisation · Environmental monitoring - European land classification * Potential natural vegetation $(\mathrm{PNV}) \cdot$ Representativity

\section{Hintergrund und Ziel}

Raumgliederungen bieten eine wichtige räumliche Bezugsgrundlage für die Überprüfung der Landschaftsrepräsentanz von Messnetzen der ökologischen Langzeitbeobachtung (Hornsmann et al. 2008; Schmidt und Schröder 2008; 
Tab. 1 Abkürzungsverzeichnis

\begin{tabular}{ll}
\hline Abkürzung & \\
\hline BfN & Bundesamt für Naturschutz \\
CART & Classification and Regression Trees \\
CHAID & Chi-square Automatic Interaction Detection \\
CRU & Climatic Research Unit \\
DFG & Deutsche Forschungsgemeinschaft \\
DHM & Digitales Höhenmodell \\
DMEER & Digital Map of European Ecological Regions \\
EC & Ecological Class, ökologische Raumklasse der ELCE \\
ELCE & Ecological Land Classification of Europe/Landschafts- \\
& ökologische Raumgliederung für Europa \\
ELCE40 & ELCE mit 40 Endklassen \\
FAO & Food and Agriculture Organization of the United Nations, \\
& Ernährung und Landwirtschaftsorganisation der Verein- \\
FK & Fen Nationen \\
GLOBE & Global Land One-kilometer Base Elevation \\
NGDC & National Geophysical Data Center \\
NN & Normal Null \\
NOAA & National Oceanic and Atmospheric Administration \\
PNV & Potenziell Natürliche Vegetation \\
PNV-Hf & PNV-Hauptformation \\
PNV-Ke & PNV-Kartierungseinheit \\
UNESCO & United Nations Educational, Scientific and Cultural \\
& Organization, \\
Organisation der Vereinten Nationen für Erziehung, \\
WebGIS & Internet-Geoinformationssystem \\
WMO & World Meteorological Organization \\
WWF & World Wide Fund for Nature \\
\hline &
\end{tabular}

Schröder und Schmidt 2000; Schröder et al. 2006). In einem von der Deutschen Forschungsgemeinschaft $\left(\mathrm{DFG}^{1}\right)$ geförderten Projekt wurde eine landschaftsökologische Raumgliederung für Europa (Ecological Land Classification of Europe, ELCE) in zwölf Varianten räumlicher Differenzierung mit dem Verfahren Classification and Regression Trees (CART) berechnet. Das Schaubild (Abb. 1) fasst die Bearbeitungsschritte zur Berechnung der ELCE zusammen.

Im ersten Teil der Beitragsserie „Ökologische Raumgliederung Europas" wurden theoretische Grundlagen zu Raumgliederungen und die statistische Ableitung der Raumgliederung betrachtet (Hornsmann et al. 2008). Insbesondere wurden die Bearbeitungsschritte 1 und 2 sowie 5 bis 9 in Abb. 1 in diesem ersten Beitrag beschrieben. Im zweiten Teil der Serie wird auf die gewählten Eingangskarten (s. Abb. 1, Schritt 1) näher eingegangen. Sie werden hinsichtlich ihrer Entstehung und geplanter Anwendungsmöglichkeiten beschrieben (Abschn. 2.1). Die Beschrei-

\footnotetext{
${ }^{1}$ Die in diesem Beitrag verwendeten Abkürzungen sind in Tabelle 1 zusammengetragen.
}

bung von Schritt 2 im ersten Artikel wird hier im Hinblick auf die Klassifizierung der nominalen Daten zur Bodenart ergänzt. Auch die Bearbeitungsschritte 3 und 4 werden kurz erläutert. Die Ausdifferenzierung der PNV (Schritt 5) wird aufgrund ihrer Bedeutung für die Ergebnisbeschreibung ebenso wie die Schritte 8 und 9 nochmals aufgegriffen (Abschn. 2.2.1, 2.2.2).

Eine Variante der Ergebnisse, die landschaftsökologische Raumgliederung für Europa mit 40 Endklassen (ELCE40), wird in diesem Artikel näher vorgestellt. In einem tabellarischen Überblick (Tabelle 2) werden beispielhaft jeweils die vorherrschende PNV und das Hauptverbreitungsgebiet von drei Endklassen (ecological classes, EC) der ELCE40 angegeben. Für diese drei EC erfolgt im Text eine detaillierte Beschreibung, die auch Bodenart, Höhe und klimatische Besonderheiten einschließt (Abschn. 3.2).

Zudem wird auf die Untersuchung der Qualität der Raumgliederung eingegangen. Ein Qualitätsmerkmal von Raumgliederungen ist, dass die Raumeinheiten hinsichtlich der ökologischen Kriterien möglichst unterschiedlich sind (Trennschärfe) (Schröder et al. 2006). Zwar deutet bereits die Beschreibung der EC auf Besonderheiten hin, eine Möglichkeit zur objektiven Überprüfung der Einhaltung der Trennschärfe stellt allerdings der statistische Test (UTest) nach Wilcoxon (1945), Mann und Whitney (1947) dar (Abschn. 2.3, 3.3 und 4.3). Er diente dazu, festzustellen, ob zwischen den Verteilungen der Höhen- und Klimadaten je Monat in jeweils zwei EC ein signifikanter Unterschied vorliegt (Bühl 2006; Sachs 2002; Schröder et al. 2006).

\section{Material und Methoden}

\subsection{Datengrundlage}

Die Berechnungsgrundlage für die ELCE bilden 48 digitale Karten zur potenziell natürlichen Vegetation (PNV) (Bohn et al. 2000, 2003), zur Bodenart (FAO 1996), zur Höhe über NN (Hastings et al. 1999) sowie Karten zur räumlichen Struktur von Temperatur, Niederschlag, relativer Luftfeuchtigkeit und Sonnenscheindauer (New et al. 2002). Diese Karten enthalten flächendeckende Informationen über nahezu die gesamte Landfläche Europas und lagen als Vektor- und Rasterkarten mit unterschiedlichen räumlichen Auflösungen und geographischen Projektionen vor (s. Abb. 1, Schritt 1 sowie Abschn. 2.2.1).

\subsubsection{Potenziell natürliche Vegetation}

Die potenziell natürliche Vegetation gilt als Integralindikator für die ökologischen Bedingungen einer Region (Hornsmann et al. 2008; Schröder 2005; Schröder et al. 2006). Aus diesem Grund gehen die Daten zur PNV als Zielvariable in 
$\mathrm{Nr}$.

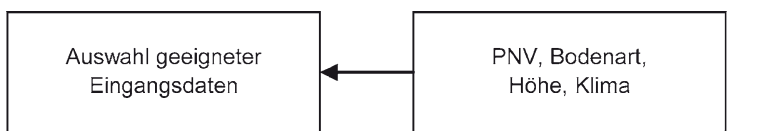

Probedurchlauf mit dem Verfahren CART

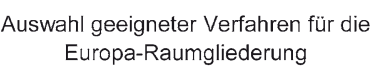

Ausdifferenzierung der PNV zur Erhöhung des Detaillierungsgrades der Ergebnisse

7

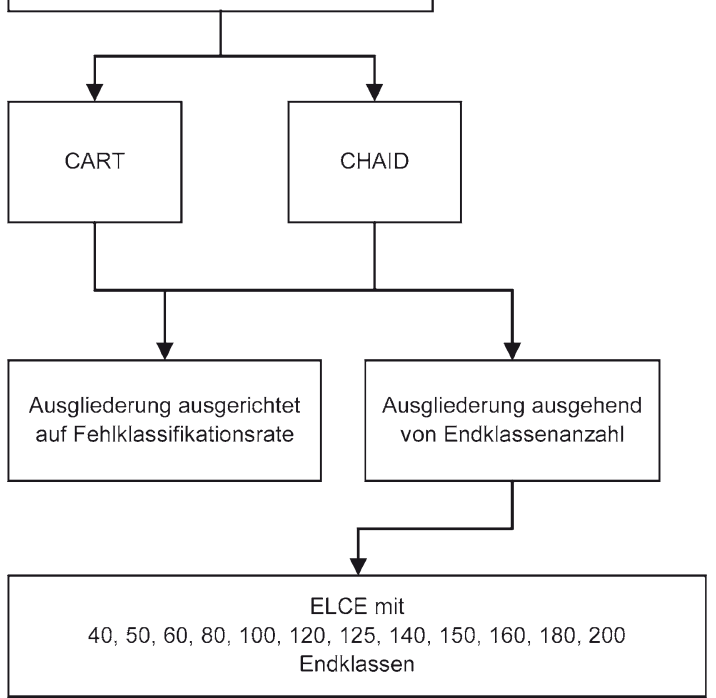

Bemerkung

Auswahl wie bei Raumgliederung Deutschlands, Baden-Württembergs, SchleswigHolsteins (Schröder et al. 2006); Ausnahmen: statt Globalstrahlung: Sonnenscheindauer; statt Verdunstung: relative Luftfeuchtigkeit, da höhere Auflösung der Karten (10 Bogenminuten statt 0,5 Grad); weitere Klimaelemente: Temperatur, Niederschlag

Umprojezierung in die Albers-Projektion;

Rasterung in $10 \times 10 \mathrm{~km}^{2}$;

Klassifizierung der nominalen Eingangsdaten PNV, Bodenart;

Auslesen der Daten als ASCII-File

Einteilung der Zielvariable PNV in 19 Klassen, orientiert an den PNV-Hauptformationen.

Raumgliederungsversionen zu undifferenziert

Basis für die neue Klassifizierung der PNV: Grundeinheiten der PNV-Karte, die PNVKartierungseinheiten; über 700 Einheiten in Europa, Verfahren CART und CHAID erlauben nur 64 Ausprägungen einer nominalen Zielvariabeln; Karte Europas wird in Teilgebiete aufgeteilt, orientiert an den PNV-Hauptformationen; zusätzliche Unterteilung der Gebiete mit den PNV-Hauptformationen " $F$ " und "G" nötig; insgesamt 24 Teilgebiete

Voraussetzungen:

Verarbeitung großer Datenmengen (bis zu 30.798 Zellen mit je 48 Ausprägungen) Daten unterschiedlicher Skalen parallel bearbeiten
Ausgliederung ausgerichtet auf Unterschreiten einer bestimmten Fehlklassifikationsrate (z.B. 0,6) verworfen;

neues Vorgehen: festgelegte Gesamtanzahl an Raumklassen flächenproportional auf Teilgebiete verteilen;

CHAID weniger geeignet als CART; u.a. nicht immer gewünschte Anzahl an Endklassen je Gebiet ableitbar

Es wurden 12 Versionen der ELCE mit CART berechnet.

Abb. 1 Bearbeitungsschritte zur Berechnung der Raumgliederung Europas (ELCE)

das CART-Verfahren (Abschn. 2.2.2) ein. Die PNV-Karte entstand in einem internationalen Projekt, das 1975 begann und im Jahr 2000 abgeschlossen wurde. Um eine aussagekräftige und vielfältig anwendbare PNV-Karte zu erhalten, war es notwendig, ein einheitliches, umfassendes und für alle Bearbeiter anwendbares Kartierungs- und Legendierungskonzept zu beschließen. Bohn et al. (2000, 2003, S. 5) beschreiben den Inhalt ihrer Karte als Wiedergabe der po- 
Tab. 2 Übersicht über die drei Europäischen Raumklassen (EC) D_14, D_22 und J 2 der Europäischen Raumgliederung mit 40 Endklassen (ELCE 40) hinsichtlich ihres Flächenanteils in Europa, ihrer Fehlklassifikation (FK), ihrer PNV-Kartierungseinheiten (PNV-Ke) mit einem Anteil von größer als 5\% an der EC sowie ihres Hauptverbreitungsgebietes (Quellen: Bohn et al. 2000, 2003, eigene Zusammenstellung)

\begin{tabular}{|c|c|c|c|c|c|}
\hline ID & $\begin{array}{l}\text { EC-Bezeich- } \\
\text { nung }\end{array}$ & $\begin{array}{l}\text { Flächen- } \\
\text { anteil in \% }\end{array}$ & FK & $\begin{array}{c}\text { PNV-Ke (Identifikation aus Bohn et al. 2000, 2003, } \\
\text { Anteil an der EC in \%) (gekürzt) }\end{array}$ & Hauptverbreitungsgebiet \\
\hline 14 & D_14 & 5,4 & 0,464 & $\begin{array}{l}\text { Krautreiche Laub-Fichtenmischwälder (D19, 54\%); } \\
\text { psammophytische Kiefernwälder mit Zwergsträu- } \\
\text { chern und krautigen Pflanzen, mit Flechten und } \\
\text { Moosen (D49, 20\%); psammophytische Kiefern- } \\
\text { wälder, teilweise mit Steppenpflanzen (D55, 12\%) }\end{array}$ & $\begin{array}{l}\text { Weißrussland: Norden; Russland: Gürtel } \\
\text { von der weißrussischen Grenze bis zur Mit- } \\
\text { telwolga, schmaler Bereich noch bis zum } \\
\text { Mittellauf der Vjatka; Litauen, Lettland, Est- } \\
\text { land; Südspitze Schwedens, Insel Gotland }\end{array}$ \\
\hline 9 & D_22 & 5,3 & 0,528 & $\begin{array}{l}\text { Moosreiche Fichtenwälder mit Zwergsträuchern } \\
\text { und Kräutern (D4, 47\%); Kiefernwälder mit Zwerg- } \\
\text { sträuchern, Flechten und Moosen (D45, 14\%); } \\
\text { Fichtenwälder mit Kräutern, Zwergsträuchern und } \\
\text { Moosen (D8, 8\%); hygrophile Fichtenwälder mit } \\
\text { Zwergsträuchern, Seggen und Moosen (D5, 6\%); } \\
\text { Fichtenwälder mit Zwergsträuchern, Kräutern und } \\
\text { Moosen im Wechsel mit Fichten- und Kiefernwäl- } \\
\text { dern auf Torfböden und mit Mooren (D6, 5\%) }\end{array}$ & $\begin{array}{l}\text { Schweden: großflächig von Värmland bis } \\
\text { Norrbotten; Russland: nordrussisches Tief- } \\
\text { land }\end{array}$ \\
\hline 29 & J_2 & 4,4 & 0,861 & $\begin{array}{l}\text { Basiphile Steineichen-Rundblättrige Eichenwälder } \\
\text { (J4, 14\%; J9, 7\%); Wildölbaum-Johannisbrotbaum- } \\
\text { wälder (J48, 7\%); Wildölbaum-Johannisbrotbaum- } \\
\text { wälder und Kermeseichen-Pistazien-Gebüsche (J49, } \\
6 \%) \text {; Steineichen- und Kermeseichenwälder (J22, } \\
5 \%)\end{array}$ & $\begin{array}{l}\text { Gebiete an der Mittelmeerküste; Innenland- } \\
\text { flächen v.a in Spanien, Portugal; Spanien: } \\
\text { Kastilien, Aragon, La Rioja, Navarra, Kata- } \\
\text { lonien, Valencia, Castilla-La Mancha, Ost- } \\
\text { Andalusien, Murcia, Sierra Nevada; Süd- } \\
\text { und Mittel-Portugal }\end{array}$ \\
\hline
\end{tabular}

tenziellen „Verbreitung der vorherrschenden natürlichen Pflanzengesellschaften, die im Einklang mit den aktuellen klimatischen und edaphischen Gegebenheiten stehen". Menschliche Eingriffe werden dabei ebenso wenig beachtet wie Änderungen, die aufgrund von Stoffeinträgen und Klimawandel entstehen könnten. Der Einfluss der geografischen Lage und der Höhe auf die Vegetation wird wiedergegeben. Die Bestimmung der PNV beruht auf „Restbeständen der natürlichen und naturnahen Ökosysteme und deren Korrelierung mit bestimmten Standortbedingungen (Klima, Boden bzw. Wärme-, Nährstoff- und Wasserhaushalt)“" (Bohn et al. 2000, 2003, S. 7). Anhand der Karte lässt sich „die Lage und Gesamtausdehnung von Gebieten mit ähnlichen Standorteigenschaften bzw. Umweltbedingungen“ nachvollziehen (Bohn et al. 2000, 2003, S. 7).

Jede Kartierungseinheit (PNV-Ke) wird durch einen Großbuchstaben, der für die 19 PNV-Hauptformationen (PNV-Hf) bzw. PNV-Komplexe steht, und einer Nummer eindeutig gekennzeichnet. Zudem werden Angaben zu den unter den heutigen ökologischen Gegebenheiten voraussichtlich vorkommenden Pflanzengesellschaften gemacht sowie zu weiteren wichtigen ökologischen Randbedingungen (Bodenart, Bodentyp und Ausgangsgestein, Geomorphologie, Klima mit Angaben zu Jahresdurchschnittswerten) und zur geografischen Lage (Bohn et al. 2000, 2003). Die Beschreibung der in den EC am häufigsten auftretenden PNV-Ke (Abschnitt 3.2, s. Tabelle 2) beruht auf den - gekürzt wiedergegebenen - Beschreibungen zur PNV und zum Verbreitungsgebiet.
Als Anwendungsbeispiele der PNV-Karte werden bei Bohn et al. $(2000,2003)$ u. a. die Ableitung von ökologischen Raumgliederungen genannt. Tatsächlich wurde die PNV-Karte bereits zur Ableitung der digitalen Karte der europäischen Ökoregionen (Digital Map of European Ecological Regions, DMEER) genutzt (Painho und Augusto 2005). Zudem diente sie als Grundlage für den europäischen Teil der terrestrischen Ökoregionen der Welt des World Wide Fund for Nature (WWF; WWF's Terrestrial Ecoregions of the World) (Morrison und Olson 2005).

\subsubsection{Höhenmodell}

Die Höhe geht aufgrund ihrer Bedeutung für die Wuchsbedingungen von Pflanzen als landschaftsökologisches Merkmal in die Berechnungen der ELCE ein (Schröder et al. 2006). Verwendet wurde das Digitale Höhenmodell (DHM) GLOBE (Global Land One-kilometer Base Elevation) vom National Geophysical Data Center (NGDC) und der National Oceanic and Atmospheric Administration (NOAA) mit einer Auflösung von 30×30 Bogensekunden oder ca. $1 \times 1 \mathrm{~km}^{2}$ (NGDC 1993). Die vor der Erstellung von GLOBE vorliegenden weltweiten DHM mit einer Auflösung von $5 \times 5$ Bogenminuten waren zu ungenau für einige Zielsetzungen dieser Organisationen, z. B. der Verarbeitung von Satellitendaten. Zwar gab es DHM in höherer Auflösung, allerdings nur von Teilgebieten der Welt. Neben diesen Karten stellte auch das von der National Imagery and Mapping Agency (NIMA) 1994 entwickelte DHM (Digital Terrain Elevation 
Data, DTED) einen wesentlichen Bestandteil von GLOBE dar. Insgesamt bildeten sechs Raster-DEMs und fünf kartographische Quellen die Grundlage für GLOBE. Aus diesen Daten wurde durch „Nearest Neighbour“ Operationen, Median-Berechnungen u. a. ein DHM in der Auflösung von 30 Bogensekunden abgeleitet und einer Qualitätskontrolle unterzogen (Hastings et al. 1999).

\subsubsection{Bodenarten}

Die Bodenart wurde aus der Digital Soil Map of the World der Food and Agriculture Organization (FAO) und der United Nations Educational, Scientific and Cultural Organization (UNESCO) abgeleitet und als landschaftsökologisches Merkmal in die Berechnung der ELCE aufgenommen. Sie gibt u. a. Aufschluss über den Wasser- und Mineralstoffhaushalt im Boden und damit auch über die Wuchsbedingungen für Pflanzen (Schröder et al. 2006).

1960 empfahl die International Society of Soil Science (ISSS) die Zusammenführung von bestehenden Bodenkarten, so dass Karten für größere Regionen oder Kontinente entstehen sollten. Daraufhin beschlossen die FAO und die UNESCO 1961, eine Bodenkarte der Welt im Maßstab von 1:5 Mio. zu entwickeln. Diese Entwicklung nahm 20 Jahre in Anspruch. In Zusammenarbeit mit Experten weltweit wurden nicht nur bestehende Karten vereinheitlicht, sondern auch systematische Felderhebungen durchgeführt. Nach und nach wurden von 1971 bis 1981 analoge Karten der Kontinente veröffentlicht (FAO 1971-1981). Die für die Ableitung der ELCE verwendete Bodenkarte (FAO 1996) resultiert aus den Bestrebungen, die analoge Karte zu digitalisieren und mit Zusatzinformationen (z. B. Bodenartenzusammensetzung) zu versehen (Nachtergaele 2002).

Weltweite Bodeninformationen dienen unter anderem als Eingangsgrößen bei Modellierungen z. B. zum Wasserhaushalt, zum Wachstum von Feldfrüchten und zur Abschätzung des Einflusses von Landnutzungspraktiken (Nachtergaele 2002).

\subsubsection{Klima}

Die mittlere monatliche Lufttemperatur, Luftfeuchtigkeit und Sonnenscheindauer sowie der mittlere monatliche Niederschlag der Jahre 1961 bis 1990 stellen wesentliche Einflussfaktoren für die Vegetation dar. Beim Klimaelement Sonnenscheindauer gehen nur die Monatsmittelwerte von März bis November als Layer in die Berechnung der ELCE ein (Abschn. 4.1).

Die Auswahl der Klimakarten orientierte sich an den bereits für die Berechnung der ökologischen Raumgliederung für Deutschland (Schröder et al. 2006) erfolgreich verwendeten Karten. Die Climate Research Unit (CRU) hat globale Klimakarten in einer Auflösung von 10 Bogenmi- nuten herausgegeben (CRU 2002; New et al. 2002). Da die Klimadaten jedoch keine Karten zur Globalstrahlung und der Verdunstung umfassen, wurden diese durch Karten zur Sonnenscheindauer und relativen Luftfeuchtigkeit ersetzt. New et al. (2002) geben mehrere Bezugs- und Datengrundlagen an, darunter die Nationalen Wetterdienste und die World Meteorological Organization (WMO) sowie zusätzliche Stationen aus weiteren globalen Datenmessnetzen, die Zeitreihen von monatlichen Klimadaten erfassen. Die Anzahl der Stationen unterscheidet sich je nach betrachtetem Klimaelement. Die verwendeten Niederschlagsdaten basieren auf 27.075 Stationen weltweit, die Informationen zur Sonnenscheindauer auf 4.792 Messpunkten und die für die Lufttemperatur auf 12.783. Für die relative Luftfeuchtigkeit wurden auch Stationen hinzugezogen, die Wasserdampfdruck gemessen haben, die dann in Werte zur relativen Feuchtigkeit umgerechnet wurden. Mit diesen Stationen standen insgesamt für dieses Merkmal 5.110 Stationen weltweit zur Verfügung (New et al. 2002).

Nach der Qualitätskontrolle führten New et al. (2002) die Interpolation der Klimadaten mit sogenannten ,thin-plate smoothing splines“ durch. Flächendeckende Daten zum Klima über größere Zeiträume werden für viele verschiedene Bereiche der Umweltwissenschaften benötigt, z. B. für die Land- und Forstwirtschaft, aber auch für Studien über Wasservorkommen und Klimaveränderungen (New et al. 2002).

\subsection{Berechnung der ELCE}

Abbildung 1 zeigt einen Überblick über die Bearbeitungsschritte zur Berechnung der ELCE. Auf einige Schritte wird im Folgenden näher eingegangen (Abschn. 1).

\subsubsection{Datenaufbereitung}

Zur statistischen Verarbeitung aller Eingangsdaten mussten diese zunächst in einem Geografischen Informationssystem (GIS) in Rasterkarten mit einer Auflösung von $10 \times 10 \mathrm{~km}^{2}$ mit flächentreuer Albers-Projektion überführt werden. Vor der Ableitung der Raumklassen mit dem CART-Verfahren (Abschn. 2.2.2) mussten für die Karten, welche die nominalen Variablen PNV und Bodenart abbilden, Klassen gebildet werden. Um die Informationen der FAO-Bodentypenkarte für die Berechnung der ELCE nutzen zu können, wurden anhand der in einer Zusatztabelle der Karte angegebenen prozentualen Kornfraktionsanteile mit Hilfe der „Bodenkundlichen Kartieranleitung" (AG Boden 1994, Tabelle 26, „Grenzwerte der Bodenarten") insgesamt 28 verschiedene Bodenartenklassen abgeleitet (s. Abb. 1, Schritt 2).

In einer ersten Version der ELCE wurde die PNV als Zielvariable in 19 Klassen anhand der PNV-Hf aufgeteilt. Dieser erste Ansatz war nicht zufriedenstellend, da das Glie- 
derungsergebnis besonders in Mitteleuropa zu undifferenziert war, da die PNV-Hf hier erst durch eine große Anzahl von Unterkategorien weiter ausdifferenziert werden. Die 19 PNV-Hf stellten daher eine zu wenig spezifizierte Ableitungsgrundlage dar. Deshalb wurden in einem zweiten Ansatz die untersten Einheiten der PNV-Karte als Zielvariable gewählt (Kartierungseinheiten: PNV-Ke) (s. Abb. 1, Schritte 3 und 4).

Da zur Anwendung des CART-Verfahrens allerdings keine Karten mit mehr als 64 nominalen Legendeneinheiten verwendet werden können, musste eine Voreinteilung der PNV-Karte, die ursprünglich über 700 PNV-Ke enthielt, vorgenommen werden (s. Abb. 1, Schritt 5). Zu diesem Zweck wurde die Karte ausgehend von den 19 PNV-Hf, die gemäß Bohn et al. $(2000,2003)$ mit Großbuchstaben abgekürzt werden, in insgesamt 24 Teilräume aufgeteilt, für die die CART-Berechnungen zunächst jeweils separat durchgeführt wurden. Anschließend erfolgte im GIS die Zusammenführung der Einzelberechnungen. Die eigentliche Anzahl von 19 Hauptformationen erhöhte sich zusätzlich, weil die PNV-Hf der Großbuchstaben F und G jeweils nochmals mehr als 64 Grundeinheiten umfassten. Sie mussten daher in Anlehnung an die Legendenunterteilung der PNV-Hf nach Bohn et al. $(2000,2003)$ wiederum in fünf bzw. zwei Klassen unterteilt werden, so dass sich insgesamt 24 Teilgebiete ergaben. Für jedes der 24 Gebiete wurden entsprechend auch die 47 Karten der beschreibenden Eingangsgrößen (Bodenart, Höhe über NN, Temperatur, rel. Luftfeuchtigkeit, Niederschlag und Sonnenscheindauer) ausgeschnitten und für die statistische Weiterverarbeitung in einem gemeinsamen ASCII-File ausgelesen, das für jede Zelle des Rasters die Koordinaten und den entsprechenden Wert der Zielvariable und der beschreibenden Variablen enthält. Für unterschiedliche Anwendungs- und Darstellungszwecke wurden 12 ELCE-Varianten mit 40, 50, 60, 80, 100, 120, 125, $140,150,160,180$ und 200 Klassen berechnet (s. Abb. 1, Schritt 9). Die zu erzielende Anzahl an Raumklassen wurde vor der Berechnung mit CART festgelegt: Da die Ableitung der Raumgliederung aufgrund des geschilderten Differenzierungsgrades der Zielvariable PNV nicht in einem Schritt durchgeführt werden konnte, musste zunächst ermittelt werden, wie viele Endklassen für die jeweilige Teilregion mit dem CART-Verfahren berechnet werden sollen. Dies wurde anhand des Kriteriums der Flächenproportionalität für jede der 24 Einzelkarten festgelegt, so dass die Gliederungstiefe für Regionen mit einem großen Flächenanteil höher ausfiel als für Klassen mit einer geringeren räumlichen Verbreitung (s. Abb. 1, Schritt 8). Die Anzahl an Raumklassen für jede Teilregion ergibt sich demnach aus der Multiplikation ihres Flächenanteils mit der jeweils festzulegenden Gesamtzahl an Raumklassen, geteilt durch 100. Für eine PNV-Hf, die einen Flächenanteil von 20\% in Europa einnimmt, wurden demnach bei einer vorgegebenen Gesamtanzahl von 50
Raumklassen 10 Endknoten bei der Berechnung des Dendrogramms (Abschn. 2.2.2) zugelassen. Für eine Aufgliederung der Teilräume durch das CART-Verfahren mussten nach Anwendung dieses Kriteriums der Flächenproportionalität mindestens zwei Raumklassen auf das Gebiet entfallen. Je nach Größe der Gebiete und der Klassenanzahl der Raumgliederung führte dieses Vorgehen allerdings bei mehreren Gebieten/PNV-Hf dazu, dass sie zu einer „Resteklasse" in der Raumgliederung zusammengefasst wurden, da sie aufgrund ihres geringen Flächenanteils (kleiner als 1,25\% und entsprechend weniger als 0,5 Raumklassen bei 40 Endklassen) ohne eigene ELCE-Klasse blieben. Andere, etwas größeren Gebiete, die im Fall der ELCE mit 40 Endklassen (ELCE40) einen Flächenanteil zwischen 1,25\% und 3,75\% ( 0,5 und 1,5 Raumklassen) besaßen, bekamen nur eine eigene Raumklasse zugewiesen und konnten daher nicht weiter untergliedert werden. Ihre Bezeichnung setzt sich aus dem Großbuchstaben der PNV-Hf und einer Null zusammen. Das größte Gebiet, das durch die PNV-Hf mit dem Buchstaben D (,mesophytische und hygromesophytische Nadelund Laub-Nadelwälder" (Bohn et al. 2000, 2003) gebildet wird, wurde bei der geringsten gewählten Endklassenanzahl von 40 Endklassen mit dem CART-Verfahren (Abschn. 2.2.2) in 12 Endklassen untergliedert. Die Benennung der mit CART abgeleiteten Klassen setzt sich aus dem Großbuchstaben (hier D) der PNV-Hf und der von CART vergebenen Nummer für die Endklassen zusammen.

\subsubsection{CART-Verfahren}

Für die Ableitung der ELCE wurde das Klassifikationsverfahren CART (Breimann et al. 1984) verwendet (Hornsmann et al. 2008; Schröder et al. 2006), das im SPSS-Programm AnswerTree 3.1 (2002) enthalten ist. CART ist ein Klassifikationsverfahren, das die Ergebnisse als hierarchisch gestufte Entscheidungsregeln in Form von Dendrogrammen (Entscheidungsbäumen) ausgibt. Die Eingangsdaten bestehen aus einer definierten Zielvariablen (Kriterium), die die Verästelung des Baumes steuert, und den beschreibenden Variablen (Deskriptoren/Prädiktoren). Das Verfahren dient zur Einteilung der Ausgangsmenge von Objekten in Klassen, die in sich möglichst homogen sind, sich aber deutlich von anderen Klassen abgrenzen (Baltes-Götz 2004, Schröder et al. 2006). Im Fall der hier beschriebenen Daten dient die PNV, genauer die PNV-Ke, als Zielvariable. CART bestimmt die häufigsten PNV-Ke im jeweiligen Verzweigungsknoten. Dann ermittelt es, mit welcher Ausprägung der beschreibenden Variablen (z. B. Temperatur im Januar) eine optimale Unterteilung der Ausgangsmenge in zwei Teilmengen erfolgen kann. Die Bedingung, die das sogenannte Splitkriterium hierfür erfüllen muss, ist, dass die Inhomogenität in den entstehenden Klassen möglichst gering ist, eine PNV-Ke in dieser Teilmenge also dominiert. CART 
berechnet für jede mögliche Splitvariable eines Knotens in zwei Tochterknoten mit einer Formel die Knoteninhomogenitäten, die als Indizes bezeichnet werden (Gini- oder alternativ Twoing-Index). Aus den Indizes für den Ausgangsknoten und die Tochterknoten berechnet CART einen Wert für die Verbesserung der Homogenität der Tochterknoten gegenüber dem Ausgangsknoten. Das Splitkriterium, das den höchsten Wert für die Verbesserung (,Improvement“) ergibt, wird ausgewählt. Dieses Vorgehen wird ggf. für die gebildeten Teilmengen, auch Tochterklassen, wiederholt. Der Grad der Verzweigung des Dendrogramms wird in diesem Fall durch den Benutzer über die minimale Anzahl an Objekten, die den Eltern- oder Tochterklassen zugeordnet werden, gesteuert. Dadurch konnte die gewünschte Anzahl an Endklassen je Teilraum berechnet werden.

Ein Maß für die Güte der Klassifikation ist die Gesamtfehlklassifikation des Baumes, die nach Abschluss der Berechnungen ausgegeben wird. Zudem lässt sich die Fehlklassifikation (FK) für jede Endklasse berechnen, indem die Menge an Rasterzellen, die nicht der häufigsten PNV-Klasse zugeordnet wurde, durch die Gesamtmenge an Rasterzellen in dieser Endklasse geteilt wird. Tabelle 2 zeigt die FK für die drei ausgewählten EC der ELCE40.

Die Kombination aus Buchstabe und Nummer in der Bezeichnung der EC (Abschn. 2.2.1) ermöglicht eine eindeutige Zuordnung zu einem sogenannten Endknoten des Dendrogramms. Dadurch kann u. a. eine Aussage darüber getroffen werden, welche Splitkriterien zu einer Abtrennung der EC von den übrigen Bereichen des Gebietes der Teilkarte führten. Abbildung 2 zeigt beispielhaft ein Dendrogramm zur Ableitung von zwei Klassen im Gebiet der PNV-Hf M. „Node 0“ im Dendrogramm ist der Ausgangsknoten bzw. die Ausgangsklasse, ,Node“ 1 (M_1) und 2 (M_2) sind die Tochterklassen; sie sind bei diesem Baum auch gleichzeitig die Endklassen. In der Spalte „Category“ der Knoten stehen die Nummern der PNV-Ke, in den Spalten daneben werden der prozentuale Anteil und die absolute Anzahl der zugeordneten Fälle angegeben. Grau unterlegte Felder zeigen die häufigste PNV-Ke des Knotens an. Zudem wird der Wert für die Vebesserung (,,improvement“) der Homogenität angegeben, der in diesem Fall auf den Gini-Indizes basiert. Das Splitkriterium, das zur Aufteilung des Ausgangsknotens in zwei Unterknoten vom CART-Verfahren auf Ebene 1 gewählt wurde, ist hier „,mayreh“, die relative Luftfeuchte im Mai. Dem Knoten M_1 sind diejenigen Objekte, d. h. Zellen des Rasters, zugeordnet, die einen Wert für die Luftfeuchtigkeit von kleiner oder gleich 58,6\% im Mai aufweisen. M_2 enthält die Fälle, die diesen Wert überschreiten.

Nach dem Auslesen der Entscheidungsregeln und ihrer automatisierten Anwendung auf die in SPSS 14 (2005) vorliegenden Datensätze ist es nach Import der Daten in ArcView 3.3 (2002) möglich, die Klassen als Karte im GIS darzustellen.



Abb. 2 Beispiel für ein Dendrogramm (Gebiet der PNV-Hauptformation $\mathrm{M}$ ) 


\subsection{U-Test}

Neben der Wahl geeigneter Eingangsgrößen ist es für die Qualität einer ökologischen Raumgliederung wesentlich, dass sich die Raumklassen statistisch signifikant voneinander unterscheiden (Kriterium der Trennschärfe). Die Unterschiede in der ökologischen Ausstattung der Raumklassen, die bei der Ergebnisbeschreibung zum Ausdruck gebracht werden, können schon ein erster Anhaltspunkt dafür sein, ob das Kriterium der Trennschärfe erfüllt ist. Durch einen statistischen Test ist es jedoch möglich zu prüfen, ob dies nur Zufall ist (Bühl 2006; Sachs 2002; Schröder et al. 2006).

Der U-Test nach Wilcoxon (1945), Mann und Whitney (1947) ist ein nicht-parametrischer, d.h. verteilungsunabhängiger, Rangsummentest. Er dient dazu zu testen, ob zwei unabhängige Verteilungen derselben Grundgesamtheit angehören. Voraussetzung für den U-Test ist, dass zwei unabhängige Zufallsstichproben von Messwerten vorliegen, die aus Grundgesamtheiten mit gleicher bzw. ähnlicher Verteilungsform stammen (Sachs 2002). Die Nullhypothese für diesen Test lautet: Die Verteilungen der zwei Stichproben sind gleich, während in der Alternativhypothese behauptet wird, dass die Verteilungen nicht übereinstimmen. Ergebniswerte sind signifikant und führen zur Ablehnung der Nullhypothese, wenn die Irrtumswahrscheinlichkeit kleiner oder gleich dem Signifikanzniveau von in diesem Fall 1\% ist (Bühl 2006; Sachs 2002).

Beim U-Test für die ELCE40 wurden alle metrischen Merkmale von jeweils zwei der 40 EC in SPSS 14 (2005) getestet. Wird die Null-Hypothese abgelehnt (ein Wert für die Signifikanz von kleiner als 0,01), so ist das Kriterium der Trennschärfe für die beiden gewählten EC hinsichtlich des betrachteten Merkmals erfüllt.

\section{4 Ökologische Eigenschaften der Raumklassen}

Die berechneten ELCE-Raumklassen können hinsichtlich der zugrunde liegenden ökologischen Daten qualitativ und quantitativ beschrieben werden: Mit Hilfe von Frequenzanalyse und Kreuztabellen wurden die jeweils häufigsten PNV-Ke und die vorherrschenden Bodenartenklassen je EC bestimmt und in Histogrammen dargestellt. Die metrischen Merkmale Höhe und Klima wurden deskriptiv statistisch ausgewertet. Während die Höhendaten mehrerer EC in Boxplots oder Histogrammen vergleichend dargestellt wurden, konnten die Klimadaten in Jahresgangkurven veranschaulicht werden. Hornsmann et al. (2008) zeigen Beispiele für diese Darstellungsmöglichkeiten. Zur Visualisierung der Temperatur- und Niederschlagsdaten sowie der Humidität oder Aridität des Klimas der drei Beispiel-EC wurden mit dem Programm GeoKLIMA2.1c von Wolfgang Hanisch Klimadiagramme nach Walter und Lieth (1960) erstellt (Hanisch 2008) (Abb. 5, 6 und 7).

\section{Ergebnisse}

Die ELCE setzt sich aus jeweils 24 Teilkarten (Abschn. 2.2.1) zusammen, die verschieden tief ausdifferenziert wurden. Sie existiert in zwölf Gliederungstiefen (40, 50, 60, $80,100,120,125,140,150,160,180$ und $200 \mathrm{EC})$.

\subsection{ELCE mit 40 Endklassen (ELCE40)}

Eine Kurzcharakterisierung am Beispiel von drei der vier größten EC der ELCE 40 hinsichtlich der Vegetation und dem Hauptverbreitungsgebiet zeigt Tabelle 2. Darin werden die häufigsten PNV-Ke je EC aufgeführt, die jeweils einen Flächenanteil von größer als $5 \%$ an der EC haben. Die anschließende Beschreibung der ökologischen Eigenschaften beschränkt sich aus Platzgründen ebenfalls auf die EC D_14, D_22 und J_2 der ELCE40 und dient zur Demonstration, wie wichtige ökologische Eigenschaften der EC zusammengefasst und visualisiert werden können. Die EC D_14 und D_22 wurden für die Beschreibung ausgewählt, um Beispielraumklassen für Nordeuropa zu zeigen. In der Beschreibung können zudem ggf. ökologische Unterschiede der nahe beieinander liegenden Gebiete, die beide aus der PNV-Hf D hervorgegangen sind, beispielhaft gezeigt werden. Mit der EC J_2 wird ein Beispiel für eine Raumklasse in Südeuropa vorgestellt.

\subsection{Beschreibung von drei EC der ELCE40}

Die Beschreibung der metrischen Merkmale, also der Höhe und der langjährigen klimatischen Monatsmittelwerte, bezieht sich auf die Mediane. Es werden Unterschiede zum Median ganz Europas bzw. zu den EC derselben PNV-Hf hervorgehoben. Zusätzlich wird mit den Klimawerten, entsprechend der Vorgehensweise von Siegmund und Frankenberg (1999), eine Typisierung des Klimas der EC vorgenommen. Mittels der Jahresdurchschnittstemperatur werden die EC einer Klimazone zugeordnet. Der sogenannte hygrische Klimatyp wird bei Siegmund und Frankenberg (1999) durch eine Gegenüberstellung der monatlichen Niederschläge und der Summe der potenziellen Landschaftsverdunstung ermittelt. Da für die EC keine Werte für die potenzielle Verdunstung vorlagen, wurden die Humidität bzw. Aridität je Monat nach De Martonne (1926) und Lauer (1952) anhand der Durchschnittswerte für Niederschlag und Temperatur bestimmt. Je nach Anzahl der humiden/ariden Monate kann eine Einteilung in vier hygrische Klimatypen vorgenommen werden. Zusätzlich werden die EC einem thermischen Klimatyp zugeordnet. Er wird durch die Einordnung der Jahresamplitude der monatlichen Durchschnittstemperaturen in vier Bereiche von hochmaritim bis hochkontinental bestimmt. Als ergänzende Möglichkeit der Klassifikation des Klimas nennen Siegmund und Frankenberg (1999) die Ein- 


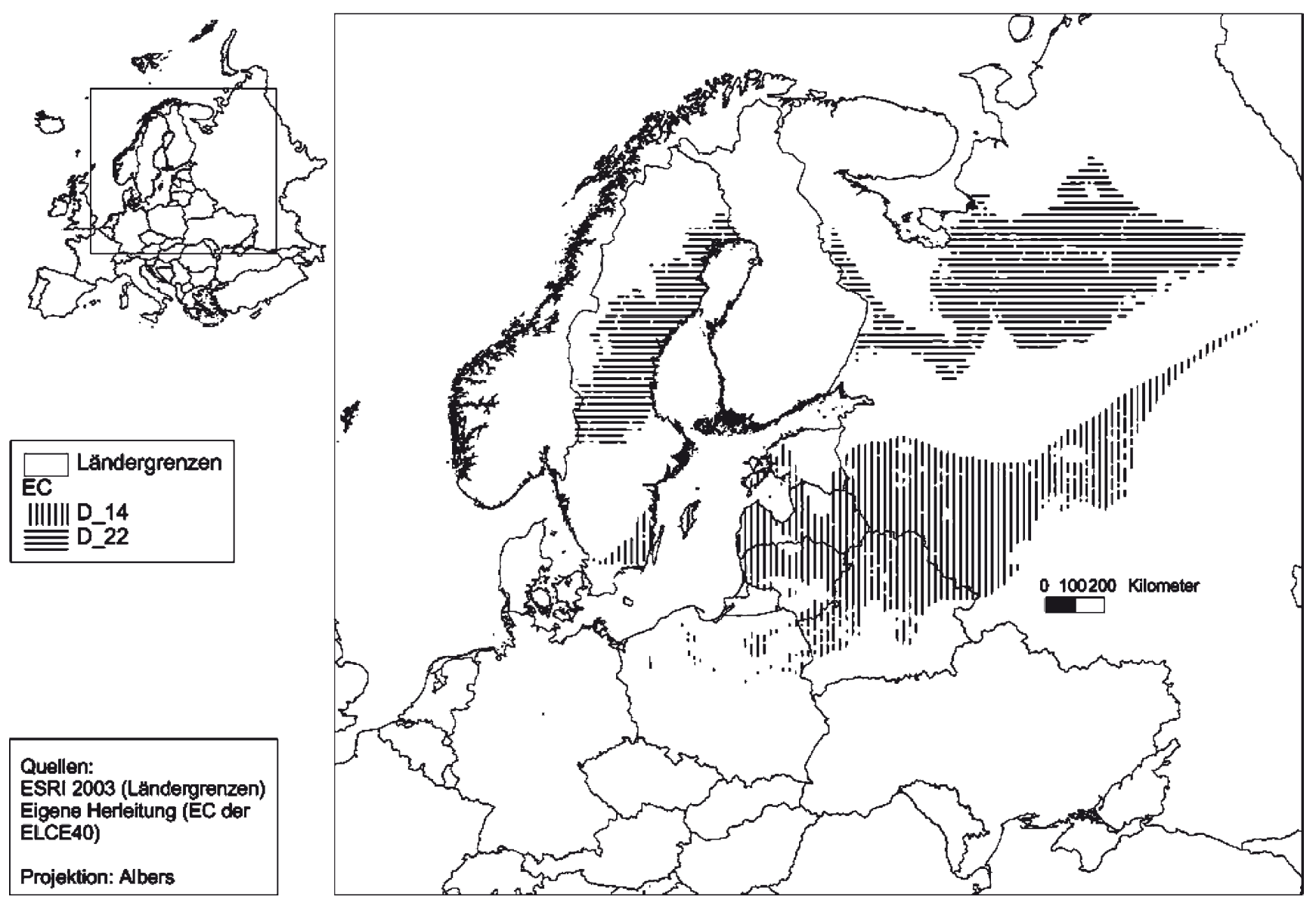

Abb. 3 Lage der Raumklassen (EC) D_14 und D_22 der Raumgliederung Europas mit 40 Endklassen (ELCE40) in Nordeuropa

schätzung der jährlichen Niederschlagsverteilung durch das Verhältnis von Sommerniederschlägen (Mai bis Oktober) zu Winterniederschlägen (November bis April). Der berechnete Prozentwert gibt Aufschluss darüber, ob das Niederschlagsaufkommen ausgeglichen ist oder Sommer- bzw. Winterregenklimate vorliegen.

Aus Platzgründen muss bei der Beschreibung der drei EC auf ausführliche Tabellen und Abbildungen verzichtet werden.

Während die Abb. 3 und 4 die Lage der EC in Europa kartografisch darstellen, zeigen die Abb. 5, 6 und 7 die Klimadiagramme der drei EC bezüglich Temperatur und Niederschlag.

\subsubsection{ECD_14}

Die größte EC der ELCE40 ist die EC D_14 mit einem Anteil von 5,4\% an der Gesamtfläche Europas. Sie ist eine der zwölf EC der Region, die durch die PNV-Hf D „Mesophytische und hygromesophytische Nadel- und Laub-Nadelwälder“ (Bohn et al. 2000, 2003, gekürzt) abgegrenzt wurde. Die EC D_14 kommt im Norden von Weißrussland vor, in Russ- land in einem Gürtel von der weißrussischen Grenze bis zur Mittelwolga und in einem schmalen Bereich bis zum Mittellauf der Vjatka. Zudem deckt die EC Teile von Litauen, Lettland und die Südspitze Schwedens sowie die Insel Gotland ab (s. Abb. 3). Die dominierende Bodenart sind Schluffe. Mit einem Flächenanteil von über 50\% wären in dieser Teilregion „baltisch-nordwest-sarmatische krautreiche LaubFichtenmischwälder“ (PNV-Ke D19) als PNV zu finden (Bohn et al. 2000, 2003, gekürzt) (s. Tab. 2). Die Höhe liegt mit durchschnittlich $147 \mathrm{~m}$ über NN nicht wesentlich unter dem gesamteuropäischen Median von $169 \mathrm{~m}$. Bei den anderen 11 EC, die das Gebiet der PNV-Hf D unterteilen, sind die Mediane zum Teil deutlich höher (EC D_7 und D_10: $291 \mathrm{~m}$ bzw. $278 \mathrm{~m}$ ) bzw. deutlich niedriger (EC D_18: $94 \mathrm{~m}$ ).

Hinsichtlich der Jahresdurchschnittstemperatur gehört die EC D_14 zur Klimazone der Mittelbreiten. Sie ist durch humides, kontinentales Klima geprägt. Die Niederschlagswerte der EC D_14 liegen nur im Juni und Juli etwa $15 \mathrm{~mm}$ höher als im europäischen Durchschnitt. Sie schwanken deutlich zwischen den Sommer- und Wintermonaten $(29 \mathrm{~mm}$ im Februar gegenüber $84 \mathrm{~mm}$ im Juli, s. Abb. 5). Die EC D_14 liegt im Bereich der Sommerregenklimate. Die Kontinenta- 



Quellen:

ESRI 2003 (Ländergrenzen)

Eigene Herleitung (EC der ELCE40)

Projektion: Albers

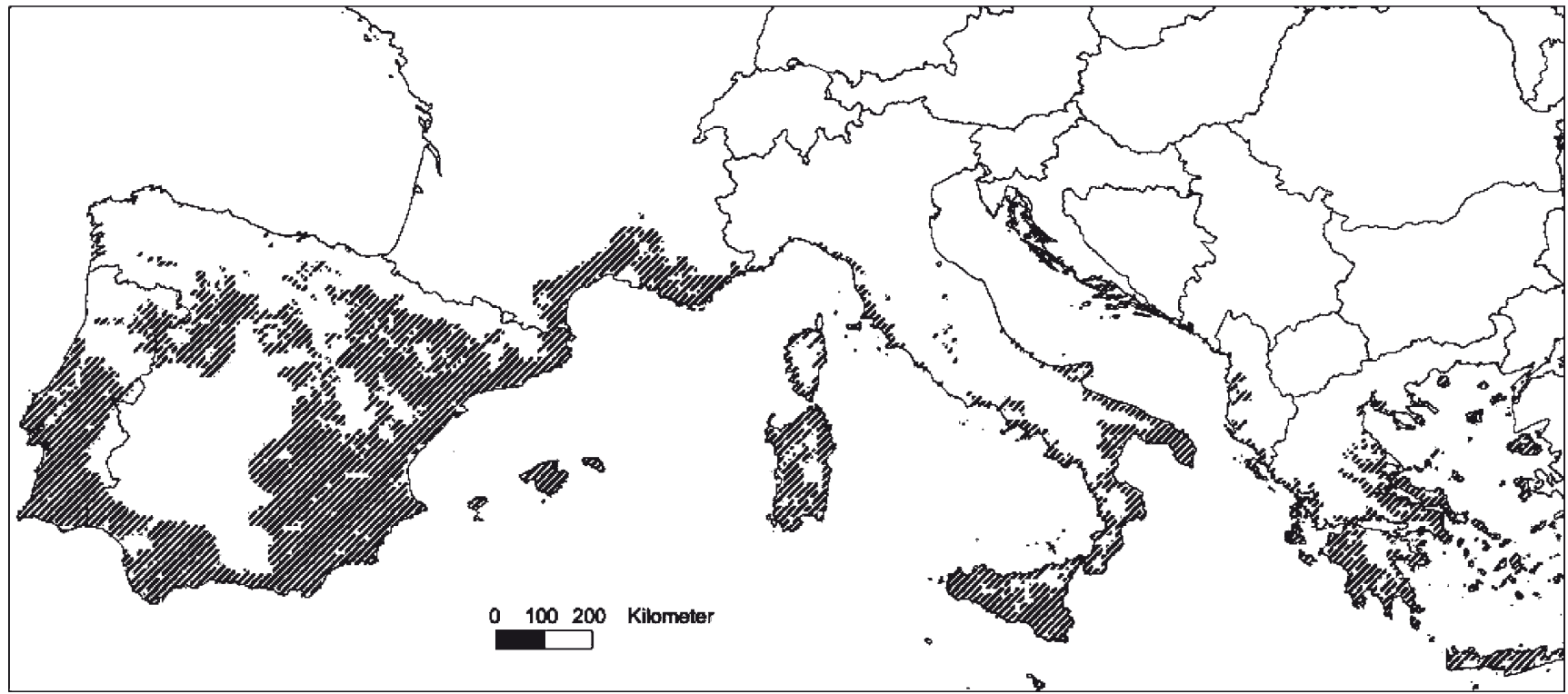

Abb. 4 Raumklasse (EC) J_2 der ELCE40 in Südeuropa

lität des Klimas der EC zeigt sich durch die Jahresamplitude der monatlichen Durchschnittstemperatur von $26^{\circ} \mathrm{C}$, denn die Durchschnittstemperatur im Jahresverlauf schwankt von $-9^{\circ} \mathrm{C}$ (Januar) bis $17^{\circ} \mathrm{C}$ (Juli) (s. Abb. 5). Im Winter ist es 2 bis $3^{\circ} \mathrm{C}$ kälter als im europäischen Mittel.

Die Sonnenscheindauer aller EC der PNV-Hf D ist in den Teilräumen der EC D_14 relativ hoch, sie schwankt zwischen 15\% (November) und 53\% (Juni) des astronomisch maximal Möglichen. Die relative Luftfeuchtigkeit schwankt mit Werten zwischen 68\% (Mai) und 96\% (Januar) nicht so stark wie z. B. in der EC D_22.

\subsubsection{ECD_22}

Die zweitgrößte EC D_22 hat einen Flächenanteil von 5,3\% in Europa. In dieser Region dominieren schluffige bis sandigschluffige Böden. Die häufigste PNV-Ke der EC D_22 ist D4: „Nordeuropäische moosreiche Fichtenwälder mitZwergsträuchern und Kräutern“ (Bohn et al. 2000, 2003, gekürzt) mit fast $50 \%$ Anteil an der EC (s. Tab. 2). Die EC kommt großflächig von Värmland bis Norrbotten in Mittel-Schweden vor sowie in Russland in Süd-Karelien mit einem Streifen nordwärts entlang der finnischen Grenze und im nordrussischen Tiefland (s. Abb. 3). Der Median der Höhenverteilung entspricht mit $166 \mathrm{~m}$ in etwa dem europäischen Durchschnitt.

Die EC D_22 liegt mit einer Jahresdurchschnittstemperatur von $1^{\circ} \mathrm{C}$ zwar noch im Bereich der Mittelbreiten, nähert sich aber schon sehr der subpolaren/polaren Zone an, die Durchschnittswerte von $0^{\circ} \mathrm{C}$ und darunter aufweist. Das Klima spiegelt im Vergleich zu ganz Europa und zur EC D_14 die nördliche Lage dieser EC wider: Die Niederschlagswerte der EC D_22 sind von Juli bis Oktober um bis $\mathrm{zu} 7 \mathrm{~mm}$ höher als im europäischen Mittel. Sie schwanken mit $28 \mathrm{~mm}$ im Februar bis zu $75 \mathrm{~mm}$ im Juli (s. Abb. 6) nicht ganz so stark wie bei EC D_14 (s. Abb. 5). Dennoch fallen auch in dieser EC die meisten Niederschläge im Sommer. Alle Monate des Jahres sind humid. Die Temperaturen liegen ganzjährig deutlich unter dem europäischen Durchschnitt, besonders im Winter betragen die Temperaturunterschiede 7 bis $8^{\circ} \mathrm{C}$. Auch gegenüber der EC D_14 zeigt die EC D_22 im Jahresverlauf durchgehend niedrigere Temperaturen, die im Dezember und Januar bis über $5^{\circ} \mathrm{C}\left(5,0^{\circ} \mathrm{C}\right.$ bzw. $\left.5.5^{\circ} \mathrm{C}\right)$ geringere Werte auf- 


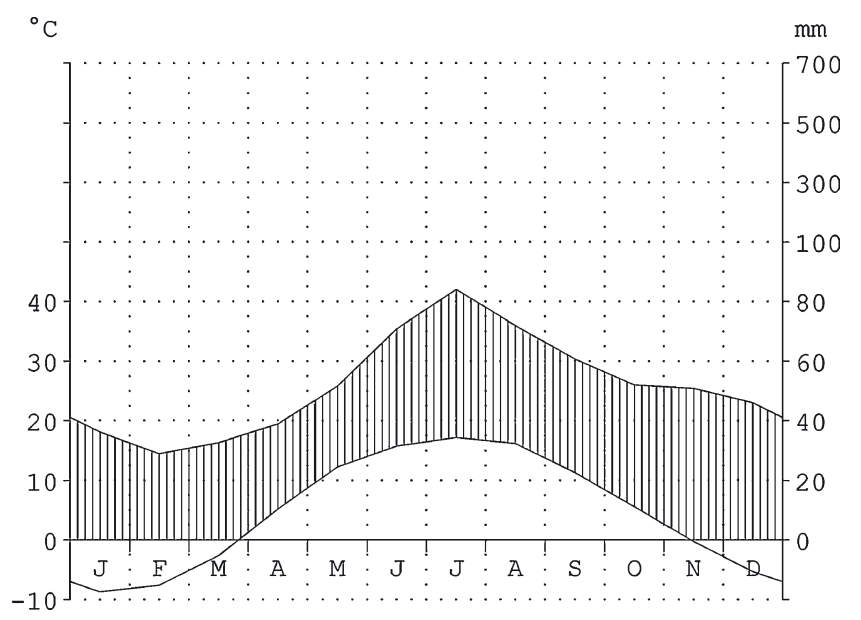

Abb. 5 Klimadiagramm nach Walter und Lieth (1960) für die Raumklasse (EC) D_14

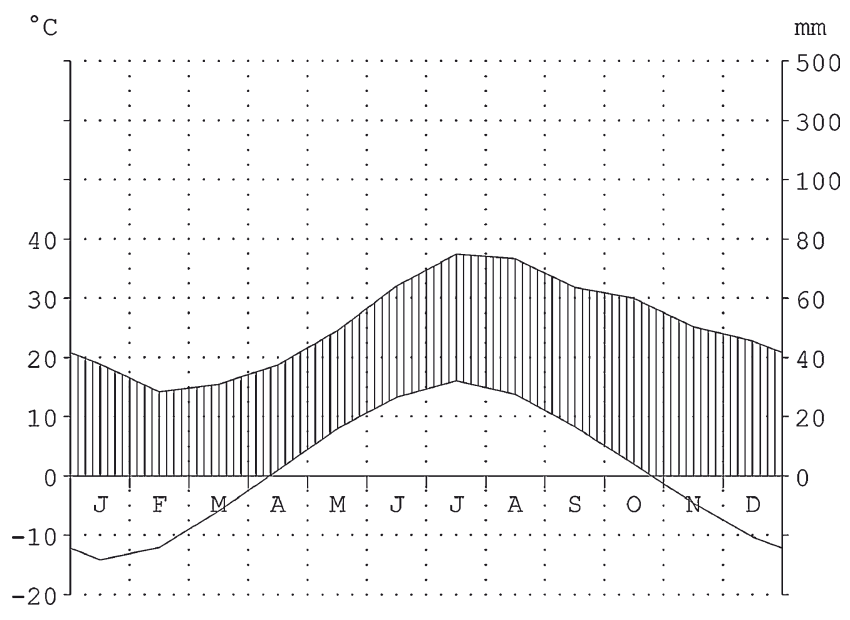

Abb. 6 Klimadiagramm nach Walter und Lieth (1960) für die Raumklasse (EC) D_22

weisen. Die Durchschnittstemperaturen (Median) schwanken im Jahresverlauf von $-14^{\circ} \mathrm{C}$ im Januar bis $16^{\circ} \mathrm{C}$ im Juli (s. Abb. 6). Das Klima der EC D_22 ist mit einer Jahrestemperaturamplitude von $30^{\circ} \mathrm{C}$ kontinental geprägt.

In den betrachteten Monaten März bis November ist die Sonnenscheindauer in der EC D_22 kürzer als in Europa und als in der EC D_14. Die Unterschiede betragen gegenüber der EC D_14 bis zu 9\% im September und Oktober und gegenüber den europäischen Durchschnittwerten in diesen Monaten sogar $13 \%$. Die relative Luftfeuchtigkeit in der EC D_22 ist von August bis April höher als in der EC D_14, sie schwankt zwischen $66 \%$ im Mai und 100\% im Januar.

\subsubsection{ECJ_2}

Mit 4,4\% Anteil an der Gesamtfläche Europas ist die EC J_2 die viertgrößte EC der ELCE40. Sie wurde für die aus-

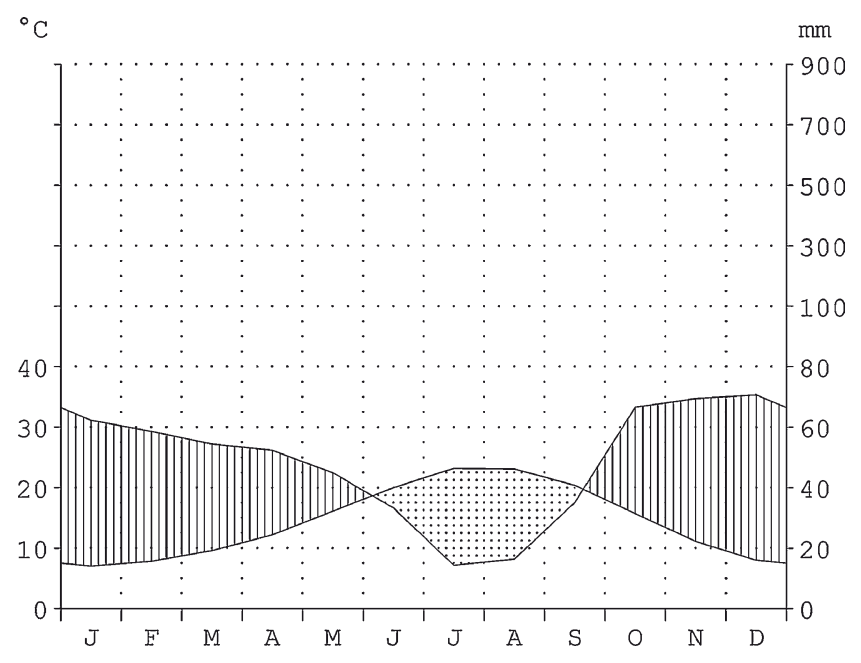

Abb. 7 Klimadiagramm nach Walter und Lieth (1960) für die Raumklasse (EC) J_2

führliche Beschreibung ausgewählt, weil sie im Unterschied zu den beiden oben beschriebenen Klassen ein Beispiel für eine EC in Südeuropa darstellt. Die Bodenart setzt sich zu $29 \%$ aus reinem Schluff, zu $21 \%$ aus stark tonigem Schluff und $\mathrm{zu} 10 \%$ aus stark schluffigem Ton zusammen. Das Gebiet der PNV-Hf J, zu der diese EC gehört, umfasst die „mediterranen Hartlaubwälder und -gebüsche“ (Bohn et al. 2000, 2003). Von den 53 PNV-Ke, die der EC J_2 zugeordnet wurden, haben 5 einen Anteil größer als $5 \%$ an dieser EC. Die PNV-Ke 4 und 9 sind mit 14\% bzw. 7\% vertreten. Diese PNV-Ke umfassen „Ost- bzw. mitteliberische mesobzw. supramediterrane basiphile Steineichen-Rundblättrige Eichen-Wälder“ (Bohn et al. 2000, 2003, gekürzt). Die PNV-Ke J48 mit einem Anteil von 7\% steht für „Mittelmediterrane Wildölbaum-Johannisbrotbaumwälder" (Bohn et al. 2000, 2003, gekürzt). „Ostmediterrane WildölbaumJohannisbrotbaumwälder und Kermeseichen-Pistazien-Gebüsche“ (J49) (Bohn et al. 2000, 2003, gekürzt) haben einen Anteil von $6 \%$ an der EC J_2. Zu rund 5\% kommen in dem Gebiet der EC zudem „Hellenisch-ägäische mesomediterrane Steineichen- und Kermeseichenwälder, z. T. im Komplex mit Wäldern aus Kalabrischen Kiefern" (J22) (Bohn et al. 2000, 2003, gekürzt) vor (s. Tabelle 2). Die EC J_2 erstreckt sich über einen weiten Streifen entlang der Mittelmeerküste und Inseln, in Spanien und Portugal ist sie auch im Binnenland verbreitet (s. Abb. 4). Die Höhe über NN ist mit einem Median von $356 \mathrm{~m}$ um fast $200 \mathrm{~m}$ höher als im europäischen Durchschnitt. Die EC J_1 derselben PNV-Hf weist jedoch mit $527 \mathrm{~m}$ einen noch höheren Median auf.

Die mediterrane Lage spiegelt sich deutlich in den Werten der Klimaelemente wider. Es liegt Mittelmeerklima vor. Die Temperaturwerte fallen ganzjährig zwischen $4{ }^{\circ} \mathrm{C}$ (Mai) und $13^{\circ} \mathrm{C}$ (Januar) höher aus als im europäischen Durchschnitt. Gegenüber der EC J_1, die sich auf das Bin- 
nenland der iberischen Halbinsel konzentriert, zeigen sich keine auffälligen Abweichungen, nur im Juni bis September liegen die Temperaturen der EC J_2 zwischen 1 und $2{ }^{\circ} \mathrm{C}$ niedriger. Mit einer Jahresdurchschnittstemperatur von $15^{\circ} \mathrm{C}$ gehört die EC J_2 zur subtropischen Klimazone. Die Temperaturamplitude beträgt $16^{\circ} \mathrm{C}$ (s. Abb. 7), das Klima ist also maritim. Die Niederschlagswerte liegen von Mai bis September bei bis zu $55 \mathrm{~mm}$ (Juli) und damit unter dem Durchschnitt, während sie in den übrigen Monaten bis zu $27 \mathrm{~mm}$ (Februar) höher ausfallen. Anders als die EC D_14 und D_22 liegt die EC J_2 im Bereich der Winterregenklimate. Die Monate Juni bis September sind arid (s. Abb. 7), insgesamt ist das Klima als semihumid einzustufen. Beim Niederschlag sind die Unterschiede zur EC J_1 nur gering ausgeprägt.

Die Sonne scheint in den Teilräumen der EC J_2 durchgehend zwischen 12 und $31 \%$ länger als im europäischen Durchschnitt. Gegenüber der EC J_1 ist die Sonnenscheindauer dagegen um bis zu $6 \%$ (August) geringer. Die relative Luftfeuchtigkeit liegt bei der EC J_2 ganzjährig zwischen 4 und $16 \%$ unter dem europäischen Durchschnitt. Hier zeigen sich auch deutliche Unterschiede zur EC J_1, deren relative Luftfeuchtigkeit in den Monaten März bis November bis zu $12 \%$ niedriger als die der J_2 ist. Die Luftfeuchtigkeit im September (J_1: 54\%, J_2: 62\%) war auch das Splitkriterium zur Aufteilung des Gebietes der PNV-Hf J in die beiden EC der ELCE 40.

\subsection{Ergebnis des U-Tests}

Die qualitativen Unterschiede in der ökologischen Ausstattung zwischen den Raumklassen können schon ein erster Anhaltspunkt dafür sein, dass das Kriterium der Trennschärfe erfüllt ist. Dies konnte durch den U-Test statistisch überprüft werden, mit dessen Hilfe alle EC-Kombinationen der ELCE40 je Merkmal auf signifikante Unterschiede getestet wurden. Es ergaben sich für $97 \%$ der Klima- und Höhendaten sehr signifikante Unterschiede der Verteilungen, die Irrtumswahrscheinlichkeit, die zur Ablehnung der Nullhypothese führt, betrug weniger als 0,01 . In der überwiegenden Mehrzahl der Fälle ist somit die Verteilung der Werte z.B. des Merkmals Höhe in EC D_7 im Vergleich zu der jeweiligen Verteilung der Ausprägung desselben Merkmals in allen anderen EC deutlich verschieden und das Kriterium der Trennschärfe erfüllt.

\section{Diskussion}

\subsection{Datengrundlagen}

Bei der PNV-Gliederung handelt es sich um eine nicht statistisch abgeleitete, sondern von Experten vollzogene
Unterteilung. Von den 19 PNV-Hf wurden laut Bohn et al. (2000, 2003) die 14 Formationen mit den Großbuchstaben A bis $\mathrm{O}$ vor allem anhand des Makroklimas, aber auch durch die Höhenstufen der Gebirge abgegrenzt. Die PNV-Hf P bis $\mathrm{U}$ stellen die azonale Vegetation dar, die anhand der Standorteigenschaften wie der Beschaffenheit der Böden und erst dann anhand des Klimas eingeteilt werden. Die weitere hierarchische Unterteilung der Formationen F und G, die aufgrund der hohen Anzahl an Kartierungseinheiten auch für die Ableitung der ELCE übernommen werden musste (Abschn. 2.2.1), orientiert sich v. a. am Mesoklima. Diese Einteilung in PNV-Hf anhand ökologischer Kriterien ist auch im Hinblick auf die Raumgliederung sinnvoll. Der Sicherung der wissenschaftlichen Qualität und Aussagekraft der PNV-Karte trugen einheitliche Richtlinien für die Kartierung und Plausibilitätskontrollen Rechnung.

Das Höhenmodell GLOBE stellt wie die FAO-Bodenkarte eine geeignete Datengrundlage dar. Die FAO-Karte ist allerdings stark generalisiert. Bei der Klassifizierung der Bodenarten konnten die in Europa vorkommenden Böden in 28 Klassen eingeteilt werden. Die häufigsten Bodenarten waren demnach reiner Schluff (Klasse 27) mit $47 \%$ und sandiger Schluff (Klasse 22) mit 15\%. Andere, inhaltlich und räumlich höher aufgelöste Bodendaten standen nicht zur Verfügung. Zwar gibt es eine Europäische Boden-Datenbank (ESDB), die kartografisch aufbereitete Informationen bezüglich der Bodenarten in Eurasien enthält. Jedoch sind diese Angaben in nur 7 Bodenartenklassen von grob bis sehr fein differenziert und waren daher als Grundlage für die ELCE nicht geeignet (ESDB 2007).

Bei den Klimadaten ist die Reduzierung der Datenmenge bei der Sonnenscheindauer auf die Monate März bis November damit zu rechtfertigen, dass die Monate Dezember bis Februar bei der Sonnenscheindauer - im Gegensatz zu den Werten der anderen Klimaelemente - keinen Einfluss auf die Ausbildung einer bestimmten Vegetationsgesellschaft ausüben (Hornsmann et al. 2008; Schröder et al. 2006). Die den Klimakarten zugrundeliegenden Interpolationsverfahren sind ebenso wie die Interpolationsfehler und die Ergebnisdiskussion bei New et al. (2002) ausführlich dokumentiert. Die Luftfeuchtigkeit wurde bei fehlenden Stationsdaten aus dem Wasserdampfdruck und der mittleren Lufttemperatur errechnet. In Schweden sind die berechneten Werte besonders im Winter höher als die direkt als Luftfeuchtigkeit gelieferten Werte in Norwegen und Finnland. New et al. (2002) führen an, dass die berechneten Werte aufgrund der niedrigen Lufttemperatur zustande kamen. Zudem lag den Daten eine sehr unterschiedliche Anzahl an Klimamessstationen zugrunde (Abschn. 2.1.4). Die Stationsdichte hat Auswirkungen auf die Qualität des Interpolationsergebnisses. Dies spiegelt bei geringer Stationsdichte, wie z.B. in Russland, weniger gut kleinräumigere Mikroklimate wider. 


\subsection{Methodik}

Das gewählte Vorgehen zur Berechnung der verschiedenen Raumgliederungsvarianten (s. Abb. 1, Abschn. 2.2) ist geeignet, die Datenfülle, die für ein differenziertes Raumgliederungsergebnis nötig ist (über 700 PNV-Ke), zu verarbeiten und die Vorgaben, die das CART-Verfahren in SPSS-AnswerTree macht (max. 64 Ausprägungen der Zielvariablen), zu erfüllen. CART ermöglicht es, die Raumklassen weitgehend bearbeiterunabhängig zu berechnen. Zudem bietet das Verfahren die Möglichkeit, aus mehreren berechneten Gliederungen anhand statistischer Maßzahlen - wie zum Beispiel der Fehlklassifikation - die statistisch optimale Gliederung zu bestimmen. Anhand des vom Verfahren ausgegebenen Dendrogramms können die Entscheidungsregeln und die Kriterien zur Ableitung für alle Raumklassen nachvollzogen werden.

Die Aufteilung Europas in 24 Teilregionen ist eine Voreinteilung durch den Bearbeiter, sie basiert aber auf der Einteilung in Legendeneinheiten der PNV-Karte, die für viele wissenschaftliche Anwendungen als geeignete Grundlage anerkannt ist (Bohn et al. 2005).

\subsection{Ergebnisse}

Die EC nehmen im Osten Europas sehr große Räume ein, während Mitteleuropa in sehr kleinräumige, nicht zusammenhängende Raumklassen eingeteilt wird. Dies liegt daran, dass die Umweltbedingungen, insbesondere Relief und Klima, in Mitteleuropa kleinräumig wesentlich stärker variieren, während sie im Osten Europas bis hin zum Uralgebirge einheitlicher sind.

Die Dendrogramme (Abschn. 2.2.2) werden hinsichtlich der vom CART-Verfahren gewählten Splitkriterien und der Ebenen, auf der diese Splitkriterien gewählt wurden, ausgewertet. Für die Ableitung der ELCE40 lagen insgesamt 10 Dendrogramme vor, die maximal 11 Splits auf 6 Verzweigungsebenen aufwiesen. Tabelle 3 enthält alle Splitkriterien, die für die Ableitung der ELCE40 von CART herangezogen wurden und die Anzahl ihres Auftretens sowie die Auflistung der Ebenen, in denen diese Kriterien gewählt wurden. Die Splitkriterien sind nach ihrer gewichteten Anzahl sortiert. Diese wurde berechnet, indem der Quotient aus der Anzahl des Auftretens des Prädiktors und der jeweiligen Splitebene, in der dieser zur Aufteilung führte, gebildet wurde. Tritt demnach ein Prädiktor einmal in der 2. Splitebene auf, beträgt der Quotient 1/2, also 0,5. Wird ein Prädiktor mehrfach auf verschiedenen Ebenen zur Teilung im Dendrogramm gewählt, wird die Summe dieser Quotienten gebildet. Das Kriterium mit der größten gewichteten Anzahl ist die Temperatur im Januar, die in drei gebildeten Bäumen jeweils gleich auf Ebene 1 das Splitkriterium war (Summe $=1 / 1+1 / 1+1 / 1=3)$.
Tab. 3 Gewichtete Splitkriterien des CART-Verfahrens der Raumgliederung Europas mit 40 Endklassen (ELCE40)

\begin{tabular}{lccc}
\hline Splitkriterien & $\begin{array}{c}\text { Anzahl } \\
\text { N }\end{array}$ & $\begin{array}{c}\text { Auftreten } \\
\text { auf Ebene }\end{array}$ & $\begin{array}{c}\text { Gewich- } \\
\text { tetes N }\end{array}$ \\
\hline Temperatur im Januar & 3 & $1,1,1$ & 3 \\
Luftfeuchtigkeit im Mai & 2 & 1,1 & 2 \\
Temperatur im Februar & 2 & 1,2 & 1,5 \\
Luftfeuchtigkeit im September & 1 & 1 & 1 \\
Niederschlag im Mai & 1 & 1 & 1 \\
Sonnenscheindauer im Oktober & 1 & 1 & 1 \\
Temperatur im April & 1 & 1 & 1 \\
Sonnenscheindauer im April & 2 & 2,3 & 0,83 \\
Sonnenscheindauer im März & 2 & 3,3 & 0,67 \\
Luftfeuchtigkeit im April & 1 & 2 & 0,5 \\
Sonnenscheindauer im November & 1 & 2 & 0,5 \\
Sonnenscheindauer im September & 1 & 2 & 0,5 \\
Temperatur im Mai & 1 & 2 & 0,5 \\
Luftfeuchtigkeit im Oktober & 1 & 3 & 0,33 \\
Luftfeuchtigkeit im November & 1 & 4 & 0,25 \\
Temperatur im November & 1 & 4 & 0,25 \\
Luftfeuchtigkeit im Februar & 1 & 5 & 0,2 \\
Niederschlag im Januar & 1 & 6 & 0,17 \\
\hline
\end{tabular}

Für die ELCE40 wurden nur Klimaelemente als Splitkriterien ausgewählt, weder die Bodenart noch die Höhe teilen die Kartierungseinheiten einer PNV-Hf so ein, dass die häufigste PNV-Ke einer PNV-Hf in einer der Tochterklassen mit einer großen Anzahl an Zellen und zusammen mit möglichst wenig anderen PNV-Ke vorkommt. Dies liegt vermutlich daran, dass bis auf das Gebiet der PNV-Hf D in den meisten Fällen nur 1 bis 2 Splits für das Erreichen der Endklassenanzahl nötig waren. Für diese erste Auftrennung der Gebiete waren Klimaelemente die entscheidenden Klassifikationskriterien, während den Boden- und vor allem auch den Höhendaten schon durch die Einteilung Europas anhand der PNV-Hf weitgehend Rechnung getragen wurde. Beispielsweise umfasst die PNV-Hf B die Gebirgsvegetation (z. B. die Rasen der Alpen, Karpaten, Pyrenäen und des Kaukasus). Die Höhenunterschiede innerhalb der einzelnen PNV-Hf sind dann bei den ersten Unterteilungen nicht so hoch, dass sie anders als einzelne Klimaelemente bei Aufteilung der Grundgesamtheit zu deutlich homogeneren Tochterklassen führen.

Die Beschreibung der ökologischen Eigenschaften der EC zeigt, dass sich benachbarte EC und auch EC, die innerhalb des Gebiets einer PNV-Hf liegen und durch die Ableitung mit CART entstanden sind, deutlich hinsichtlich der ökologischen Eigenschaften unterscheiden. Der U-Test bewies für die überwiegende Mehrheit der Kombinationen von metrischen Merkmalen signifikante Unterschiede in der Verteilung, in nur 3\% der Fälle konnten keine signifikanten Unterschiede zwischen den Prädiktoren zweier EC festgestellt werden. 
Der U-Test reagiert besonders auf Unterschiede bei den Medianen (Sachs 2002). In den vereinzelten Fällen, die vom U-Test als nicht signifikant deklariert werden, liegen dennoch Unterschiede zwischen den Eingangsgrößen der EC vor, die sich allerdings in anderen Kennwerten als dem Medianwert manifestieren.

\section{Schlussfolgerungen}

Die Eingangskarten zur Berechnung der ELCE haben sich trotz der Einschränkung insbesondere bei den Bodendaten bewährt. Mit dem CART-Verfahren ist es gelungen, ökologische Raumklassen zu kartieren und Europa auf unterschiedlichen, frei wählbaren Differenzierungsniveaus (hier: 40 bis zu 200 EC) anhand der potenziell natürlichen Vegetation mit einem Regel basierten Verfahren zu untergliedern. Jede der $10 \mathrm{~km} \times$ $10 \mathrm{~km}$ Rasterzellen lässt sich detailliert statistisch beschreiben und aufgrund der landschaftsökologischen Fundierung der Methodik (Allen und Starr 1982; Bohn et al. 2000, 2003; Klijn und de Haes 1994; Mabbutt 1968; Müller-Hohenstein 1981; Ruxton 1968; Schröder und Pesch 2005; Schröder et al. 2006; Schultz 2000; Tüxen 1956; Walter und Breckle 1999) gut interpretieren. Dies ist insofern wichtig, als ökologische Raumgliederungen im Umweltmonitoring für die Analyse und Planung von Messnetzen, die räumliche Verallgemeinerung punktuell erhobener Umweltdaten sowie für dynamische (Global Change) Modellierungen eingesetzt werden sollen (Ferretti 2001; Miller et al. 2004; Moog et al. 2004; Turner et al. 1989). Die Ergebnisse des U-Tests belegen am Beispiel der ELCE40 für alle Raumklassenkombinationen eine deutlich unterschiedliche ökologische Ausstattung und somit die Erfüllung des Qualitätskriteriums der Trennschärfe.

\section{Empfehlungen}

Die ELCE stellt, obwohl der Vergleich zu weiteren Gliederungen noch aussteht, zum jetzigen Zeitpunkt eine geeignete Grundlage für unterschiedliche Anwendungsbereiche dar, z.B. für die Repräsentanzanalyse verschiedener europäischer Umweltbeobachtungsmessnetze.

Eine erneute Berechnung sollte in Erwägung gezogen werden, sobald ein Verfahren entwickelt wird, das die Klassifikation einer nominalen Variablen mit mehr als 64 Ausprägungen erlaubt. Dadurch könnte das Einwirken des Bearbeiters insofern minimiert werden, dass beispielsweise keine Voreinteilung Europas auf Basis der PNV-Hf notwendig ist. Zudem ist, sobald höher aufgelöste und aktuellere Karten für die ökologischen Eigenschaften (z. B. veränderte klimatische Verhältnisse infolge der globalen Klimaerwärmung) für Europa vorliegen, über eine erneute Berechnung nachzudenken.

\section{Ausblick}

Der im letzten und in diesem Beitrag vorgestellten Berechnung und Qualitätsüberprüfung der europäischen Raumgliederung soll im kommenden Beitrag die Gegenüberstellung mit bereits vorhandenen Gliederungen folgen. Zudem steht auch die beispielhafte Anwendung der Raumgliederung für die Repräsentanzanalyse ausgewählter europaweiter Umweltmonitoring-Messnetze in einem weiteren Artikel im Mittelpunkt.

\section{Literatur}

ArcView 3.3 (Computer-Software) (2002) ESRI. Redlands

AG Boden (1994) Bodenkundliche Kartieranleitung (Hrsg) Bundesanstalt für Geowissenschaften und Rohstoffe und Geologische Landesämter, 4. Aufl., Hannover

Allen JM, Starr TB (1982) Hierarchy: Perspectives for ecological complexity. University of Chicago Press, Chicago

AnswerTree 3.1 (Computer-Software) (2002) SPSS Inc. Chicago

Baltes-Götz B (2004) Entscheidungsbaumanalyse mit AnswerTree 3.1. Universitätsrechenzentrum Trier. http://www.uni-trier.de/fileadmin/ urt/doku/at/at31.pdf (Zugriff am: 21.10.2008)

Bohn U, Neuhäusl R, Gollub G, Hettwer C, Neuhäuslová Z, Schlüter $\mathrm{H}$, Weber H $(2000,2003)$ Karte der natürlichen Vegetation Europas/Map of the natural vegetation of Europe. Maßstab/Scale 1:2.500.000. Teil 1 Erläuterungstext mit CD-ROM, Teil 2 Legende, Teil 3 Karten. Landwirtschaftsverlag, Münster

Bohn U, Hettwer C, Gollub G (Hrsg) (2005) Anwendung und Auswertung der Karte der natürlichen Vegetation Europas./Application and analysis of the map of the natural vegetation of Europe. Bonn, BfN-Scripten (Bundesamt für Naturschutz), S. 156

Breiman L, Friedman JH, Olshen RA, Stone CJ (1984) Classification and Regression Tree. Chapman \& Hall, Belmont, CA

Bühl A (2006) SPSS 14. Einführung in die moderne Datenanalyse. 10. Auflage. Pearson Studium, München

CRU (2002) Global Climate dataset. http://www.cru.uea.ac.uk/cru/ data/hrg.htm (Zugriff am: 21.10.2008)

ESDB (2007) European Soil Database. http://eusoils.jrc.it/ESDB Archive/ESDB/index.htm (Zugriff am: 25.1.2008)

FAO (1996) The digitized soil map of the world including derived soil properties. CD-ROM. FAO, Rome

FAO (1971-1981) The FAO-UNESCO Soil Map of the World. Legend and 9 volumes. UNESCO, Paris

Ferretti M (2001) Ecosystem monitoring. From the integration between measurements to the integration between networks. Publicazione del Corso di Cultura in Ecologia, Università degli studi di Padova, pp 3-54

Hanisch W (2008) GeoKLIMA 2.1c für Windows 95, 98, NT und XP zum Erstellen von Klimadiagrammen nach Walter/Lieth. htttp.www.w-hanisch.de/geoklima

Hastings DA, Dunbar PK, Elphingstone GM, Bootz M, Murakami H, Maruyama H, Masaharu H, Holland P, Payne J, Bryant NA, Logan TL, Muller J-P, Schreier G, MacDonald JS (eds) (1999) The Global Land One-kilometer Base Elevation (GLOBE) digital elevation model, Version 1.0. National Oceanic and Atmospheric Administration, National Geophysical Data Center, U.S.A. Digital data base on the World Wide Web. http://www.ngdc.noaa.gov/ $\mathrm{mgg} / \mathrm{topo} / \mathrm{globe}$.html (Zugriff am: 5.2.2008)

Hornsmann I, Schmidt G, Schröder W (2008) Berechnung einer landschaftsökologischen Raumgliederung Europas - Teil 1. UWSF Z Umweltchem Ökotox 20(1):25-35 
Klijn F, de Haes HAU (1994) A hierarchical approach to ecosystems and its applications for ecological land classification. Landscape Ecol 9:89-104

Lauer W (1952) Humide und aride Jahreszeiten in Afrika und Südamerika und ihre Beziehung zu den Vegetationsgürteln. Bonner geogr Abh 9:15-98

Mabbutt JA (1968) Review of concepts of land evaluation. In: Stewart GA (ed) Land evaluation. Macmillan, Melbourne, pp 11-28

Mann H, Whitney D (1947) On a test of whether one of two random variables is stochastically larger than the other. Annals of Mathematical Statistics 18:50-60

De Martonne E (1926) Une nouvelle fonction climatologique: l'indice d'aridité. La Météorologie 10:449-459

Miller JR, Turner MG, Smithwick EA, Dent CL, Stanley EH (2004) Spatial extrapolation. The science of predicting ecological patterns and processes. BioSci 54:310-320

Moog O, Schmidt-Kloiber A, Ofenbock T, Gerritsen J (2004) Does the ecoregion approach support the typological demands of the EU Water Framework Directive? Hydrobiologia 516:21-33

Morrison J, Olson DM (2005) The natural vegetation map of Europe: A regional source for WWF's terrestrial ecoregions of the world. In: Bohn U, Hettwer C, Gollub G (Hrsg) Anwendung und Auswertung der Karte der natürlichen Vegetation Europas/Application and analysis of the map of the natural vegetation of Europe. Bonn, BfN-Scripten (Bundesamt für Naturschutz) 156:71-80

Müller-Hohenstein K (1981) Die Landschaftsgürtel der Erde, 2. Auflage. Teubner Verlag, Stuttgart

Nachtergaele FO (2002) From the soil map of the world to the digital global soil and terrain database: 1960-2002. http://www.itc. nl/ rossiter/Docs/WRB/SoilMapWorld.pdf (Zugriff am: 5.2.2008)

New M, Lister D, Hulme M, Makin I (2002) A high-resolution data set of surface climate over global land areas. Clim Res 21:1-25

NGDC (1993) Global Ecosystems Database, Version 2.0. http:// www.ngdc.noaa.gov/ecosys/fliers/se-2006.shtml (Zugriff am: 21.10.2008)

Painho M, Augusto G (2005) A Digital Map of European Ecological Regions. In: Bohn U, Hettwer C, Gollub G (Hrsg) Anwendung und Auswertung der Karte der natürlichen Vegetation Europas./Application and analysis of the map of the natural vegetation of Europe. Bonn, BfN-Scripten (Bundesamt für Naturschutz) 156:27-36
Ruxton BP (1968) Order and disorder in land. In: Stewart GA (ed) Land evaluation. Papers of a CSIRO symposium. Organized in cooperation with UNESCO, 26-31 August 1968. Melbourne, pp 29-39

Sachs L (2002) Angewandte Statistik. Anwendung statistischer Methoden. 10. Auflage. Springer, Berlin

Schmidt G, Schröder W (2008) Ökologische Raumgliederung Europas - Vorwort. UWSF - Z Umweltchem Ökotox 20(1):23-24

Schröder W (2005) Die Potentielle Natürliche Vegetation als Datengrundlage einer ökologischen Raumgliederung. In: Bohn U, Hettwer C, Gollub G (Hrsg) Anwendung und Auswertung der Karte der natürlichen Vegetation Europas/Application and analysis of the map of the natural vegetation of Europe.Bonn BfN-Scripten (Bundesamt für Naturschutz) 156:37-54

Schröder W, Pesch R (2005) Geographische Umweltmessnetzanalyse und -planung. Geogr Helv 60:77-86

Schröder W, Schmidt G (2000) Raumgliederung für die Ökologische Umweltbeobachtung des Bundes und der Länder. Umweltwiss Schadst Forsch 12:237-243

Schröder W, Schmidt G, Hornsmann I (2006) Landschaftsökologische Raumgliederung Deutschlands. In: Fränzle O, Müller F, Schröder W (Hrsg) Handbuch der Umweltwissenschaften. Grundlagen und Anwendungen der Ökosystemforschung, Kap. V-1.9, 16. Erg.Lfg. ecomed, Landsberg am Lech, S 1-101

Schultz J (2000) Handbuch der Ökozonen. UTB, Stuttgart

Siegmund A, Frankenberg P (1999) Klimatypen der Erde. Ein didaktisch begründeter Klassifikationsversuch. GR 51:494-499

SPSS 14 (Computer-Software) (2005) SPSS Inc., Chicago

Tüxen R (1956) Die heutige potentiell natürliche Vegetation als Gegenstand der Vegetationskartierung. Angew Pflanz Soz 13: 5-43

Turner MG, Dale VH, Gardner RH (1989) Predicting across scales. Theory development and testing. Landscape Ecol 3:245-252

Walter H, Breckle SW (1999) Vegetation und Klimazonen, 7. Auflage. UTB, Stuttgart

Walter H, Lieth H (1960) Klimadiagramm-Weltatlas: etwa 8000 Klimastationen (etwa 9000 Diagramme), 33 Hauptkt., 22 Nebenkt. G. Fischer VEB, Jena

Wilcoxon F (1945) Individual comparisons by ranking methods. Biometrics Bull 1:80-83 